\section{Indução de endividamento hospitalar na compra de medicamento em situação de monopólio: o caso do mesilato de imatinibe}

\author{
Induction of hospital indebtedness due to \\ medicine purchases under monopoly conditions: \\ the case of imatinib mesylate
}

\author{
Inducción de la deuda hospitalaria en la compra \\ de medicamentos en monopolio: el caso de \\ mesilato de imatinib
}

Carolinne Thays Scopel 1 Gabriela Costa Chaves 1

\footnotetext{
${ }_{1}$ Escola Nacional de Saúde Pública Sergio Arouca Fundação Oswaldo Cruz, Rio de Janeiro, Brasil.

Correspondência C. T. Scopel

Núcleo de Assistência

Farmacêutica, Escola Nacional de Saúde Pública Sergio Arouca, Fundação Oswaldo Cruz.

Rua Leopoldo Bulhões 1480, Rio de Janeiro, $R J$

21041-210, Brasil. carol.thays@gmail.com
}

\begin{abstract}
Medicine expenditures consume a large share of the health budget, so knowledge on the use of these funds is essential for decision-making in public health and improvement of pharmaceutical care. This study analyzed the indebtedness of a high-complexity university hospital due to increased spending on imatinib mesylate. The descriptive study was based on analysis of documents and records in the Hospital Information System (SIH) from 2002 to 2010. Starting with inclusion of the medicine in the budget, the study mapped strategies by the pharmaceutical industry and government, as well as government responses to reduce the product's price. The systematization and publication of information stored in files and electronic databases can help monitor the results of programs funded by the Brazilian Ministry of Health.
\end{abstract}

Hospital Costs; Intellectual Property; Pharmaceutical Preparations

\section{Resumo}

Os gastos com medicamentos correspondem a uma grande parcela do orçamento em saúde. Sendo assim, a produção de conhecimento sobre o uso desses recursos é essencial na tomada de decisão em saúde pública e melhoria da assistência farmacêutica. Este estudo teve como objetivo analisar o processo de endividamento em um hospital universitário de alta complexidade devido ao gasto crescente com a aquisição de mesilato de imatinibe. Por meio de análise documental e registros no Sistema de Informações Hospitalares (SIH) entre 2002 e 2010, realizou-se um estudo descritivo. A partir do caminho da incorporação do medicamento, foram mapeadas as estratégias da indústria farmacêutica e do governo, assim como as respostas governamentais de redução do preço. A sistematização e publicação de informações guardadas em arquivos e na memória podem contribuir para o acompanhamento dos resultados dos programas mantidos pelo Ministério da Saúde.

Custos Hospitalares; Propriedade Intelectual; Preparações Farmacêuticas 


\section{Introdução}

Os crescentes gastos com medicamentos demandam uma alocação eficiente de recursos em saúde, estabelecendo um campo importante nas discussões de políticas públicas. A situação do Sistema Único de Saúde (SUS) é caracterizada por limitações na utilização dos recursos, demanda por incorporação de novas tecnologias, e interesses privados movidos pela lógica empresarial de maximização de lucros e não pelas necessidades sanitárias da população 1,2,3.

A produção de conhecimento sobre o uso de recursos com medicamentos no ambiente hospitalar constitui uma importante ferramenta para monitorar e subsidiar a tomada de decisão em saúde pública 1. Com o surgimento de novas tecnologias, aumento dos gastos em saúde e mudanças no perfil epidemiológico, diagnóstico e terapêutico, percebe-se uma desarticulação entre produção, incorporação e utilização de tecnologias no SUS 2. No caso dos hospitais públicos, a incorporação de tecnologias se soma a outros desafios da administração pública em contexto de crise e de subfinanciamento 4 .

O mesilato de imatinibe foi incorporado no SUS em 2001, como segunda linha no tratamento de leucemia mieloide crônica (LMC) 5,6. De 2002 a 2010, esse medicamento foi adquirido pelos hospitais brasileiros. Após problemas de aumento de preço, não cumprimento de acordos ou na codificação do procedimento, o Ministério da Saúde acordou com a indústria farmacêutica a redução progressiva do preço do medicamento mediante isenção de impostos, compra centralizada e distribuição pelas Secretarias Estaduais de Saúde, gerando uma economia informada de aproximadamente $\mathrm{R} \$ 400$ milhões em 2011 5,7. Esse panorama nacional pode ser verificado com base na experiência do Hospital de Clínicas da Universidade Federal do Paraná (HC-UFPR).

Este artigo objetiva analisar o processo de endividamento do HC-UFPR devido ao gasto crescente com a aquisição do mesilato de imatinibe para LMC.

\section{Metodologia}

Trata-se de um estudo descritivo da sistematização de um caso sobre os gastos na compra de um medicamento em um hospital universitário federal de atenção em nível terciário, no período de 2002 a 2010. A escolha do período da pesquisa considerou o início da aquisição do medicamento pelo hospital até a compra centralizada pelo Ministério da Saúde.
O estudo foi composto das seguintes etapas metodológicas, realizadas de forma concomitante: revisão da literatura, análise documental no HC-UFPR, sistematização das informações e análise crítica dos dados.

Foram obtidos valores mensais de utilização, custos de aquisição do mesilato de imatinibe e ressarcimento pelo SUS ao hospital por meio do registro da dispensação do medicamento pela farmácia no Sistema de Informação Hospitalar (SIH). Esses dados foram comparados com as informações contidas nos processos de compras do medicamento e no registro manual da liberação da Autorização de Procedimento de Alta Complexidade (APAC), instrumento para a cobrança do procedimento. Essas três fontes, obtidas junto aos serviços de faturamento, abastecimento e compras do hospital, foram cruzadas para corrigir eventuais erros na computação das informações.

Os dados foram sistematizados em planilhas eletrônicas. Em cada ano foram calculados: o número de pacientes em tratamento, o gasto hospitalar na compra do medicamento, o repasse financeiro do Ministério da Saúde para o HCUFPR e a diferença entre o valor repassado e o valor pago pelo hospital. Também foi calculado o custo mensal do tratamento em cada fase da doença. Foi obtida a proporção de discordância entre as diferentes fontes, a fim de monitorar os valores divergentes para serem reconferidos.

Foi contabilizada a porcentagem de aumento do preço do tratamento ao longo dos anos e a diminuição posterior à compra centralizada do medicamento. Para subsidiar a análise, foi feito um levantamento para a caracterização do hospital. Além disso, buscou-se descrever as estratégias empresariais e a resposta governamental na resolução do processo de endividamento.

Este trabalho é parte de um projeto de pesquisa mais abrangente, aprovado pelo Comitê de Ética em Pesquisa do HC-UFPR em 21 de maio de 2012 com o no CAAE 00888812.9.0000.0096.

\section{Resultados}

Entre 2002 e 2010, o número de usuários atendidos e que retiravam o medicamento no HC-UFPR apresentou um crescimento de 43 para 138. Em 2011, houve uma leve queda no número de usuários para 115. Os valores pagos na compra do mesilato de imatinibe e os repasses do Ministério da Saúde por meio da APAC também aumentaram ao longo do tempo, até abril de 2011 quando teve início a compra centralizada pelo Ministério da Saúde. Essa evolução pode ser vista na Tabela 1. 
Evolução do número de pacientes, gasto hospitalar, repasse mediante Autorização de Procedimento de Alta Complexidade (APAC) em Reais (R\$) e relação percentual entre o valor repassado e o gasto de 2002 a 2010. Os valores de gasto hospitalar e repasse por meio da APAC foram corrigidos pelo Índice Nacional de Preços ao Consumidor Amplo (IPCA).

\begin{tabular}{cccc}
\hline & Número de pacientes & Gasto hospitalar (R\$) & $\begin{array}{c}\text { Repasse por APAC (R\$) } \\
\text { Repasse em relação } \\
\text { ao gasto (\%) }\end{array}$ \\
\hline 2002 & 43 & $1.876 .535,56$ & $1.742 .547,90$ \\
2003 & 66 & $3.939 .076,61$ & $3.925 .480,51$ \\
2004 & 92 & $5.590 .972,95$ & $5.635 .135,77$ \\
2005 & 121 & $7.036 .534,59$ & $7.080 .221,35$ \\
2006 & 116 & $8.445 .946,46$ & $7.876 .637,90$ \\
2007 & 128 & $8.692 .602,39$ & $7.107 .776,99$ \\
2008 & 132 & $9.272 .789,06$ & $7.339 .420,83$ \\
2009 & 136 & $9.027 .928,72$ & 700,79 \\
2010 & 138 & $7.838 .630,89$ & $7.018 .723,70$ \\
\hline
\end{tabular}

A defasagem mensal por tratamento entre o valor pago e aquele repassado por meio da APAC em cada fase da doença está ilustrada na Figura 1 . As fases crônica, acelerada e blástica indicam a progressão da doença e são tratadas com diferentes dosagens de mesilato de imatinibe. Para o tratamento de um mês da fase crônica são necessários quatro comprimidos por dia (total de $400 \mathrm{mg} / \mathrm{dia}$ ), e nas fases acelerada e blástica são necessários, respectivamente, 6 e 8 comprimidos por dia. Essa variação na dosagem reflete não só o custo do tratamento por mês como também o valor do repasse que era feito pelo Ministério da Saúde, conforme Tabela 2.

Antes de 2006, esse déficit era notado somente para o uso na fase blástica da doença. A partir desse ano, observa-se um crescimento da dívida acumulada do HC-UFPR devido ao aumento autorizado do preço do medicamento sem a necessária atualização dos valores repassados pelo governo.

Na Figura 2, está representado o valor total da dívida por ano. Em 2009, o déficit nas compras com o mesilato de imatinibe para o tratamento de LMC passou de 1,8 milhão de reais. A dívida acumulada no período de 2002 a 2010 chegou a $\mathrm{R} \$ 6.561 .774,16$.

A Figura 3 mostra a evolução do custo mensal do tratamento na fase crônica $(400 \mathrm{mg} / \mathrm{dia})$. O primeiro aumento aconteceu em 2006, seguido por 2007 e 2008. De 2005 a 2010 esse aumento foi de 23,56\%. Em 2011, com a compra centralizada, a redução do preço do medicamento foi de mais de $50 \%$.

\section{Discussão}

\section{Estratégia de incorporação}

O HC-UFPR foi o primeiro centro público a tratar portadores de LMC como parte do protocolo de acesso expandido do medicamento ${ }^{8}$. Em 2001, foram incluídos aproximadamente 100 usuários, o que representava metade do número incluído no Brasil inteiro. Já nessa época, a melhora clínica dos pacientes com o uso do medicamento era expressiva, levando a uma expectativa muito positiva em relação à efetividade do mesmo para o tratamento da LMC.

Após o estudo de fase III que avaliou a resposta ao mesilato de imatinibe em LMC, protocolos de acesso expandido foram lançados por todo o mundo, inclusive no Brasil 9: a Novartis (São Paulo, Brasil) fornecia gratuitamente o medicamento para o maior número de pacientes, consolidando uma demanda crescente para essa nova abordagem terapêutica. A ampliação da utilização do mesilato de imatinibe acarretou um aumento expressivo de gastos para o sistema de saúde.

Segundo resolução em vigor à época, o acesso expandido refere-se à possibilidade de disponibilização de um produto novo e ainda sem registro na Agência Nacional de Vigilância Sanitária (ANVISA) mediante três condições: se o produto estiver em estudo de fase III no Brasil; ou se produto estiver em estudo de fase III e com programa de acesso expandido no país de origem; ou se o produto estiver registrado no país de origem e for indicado para situações graves ou como única alternativa terapêutica disponível no Brasil sem ônus para o paciente 10 . 
Diferença (em Reais) entre o valor repassado pelo Ministério da Saúde e o valor pago na compra do mesilato de imatinibe para um tratamento de leucemia mieloide crônica, por mês e por fase da doença, de 2002 a 2010 no Hospital de Clínicas da Universidade Federal do Paraná (HC-UFPR), Brasil.

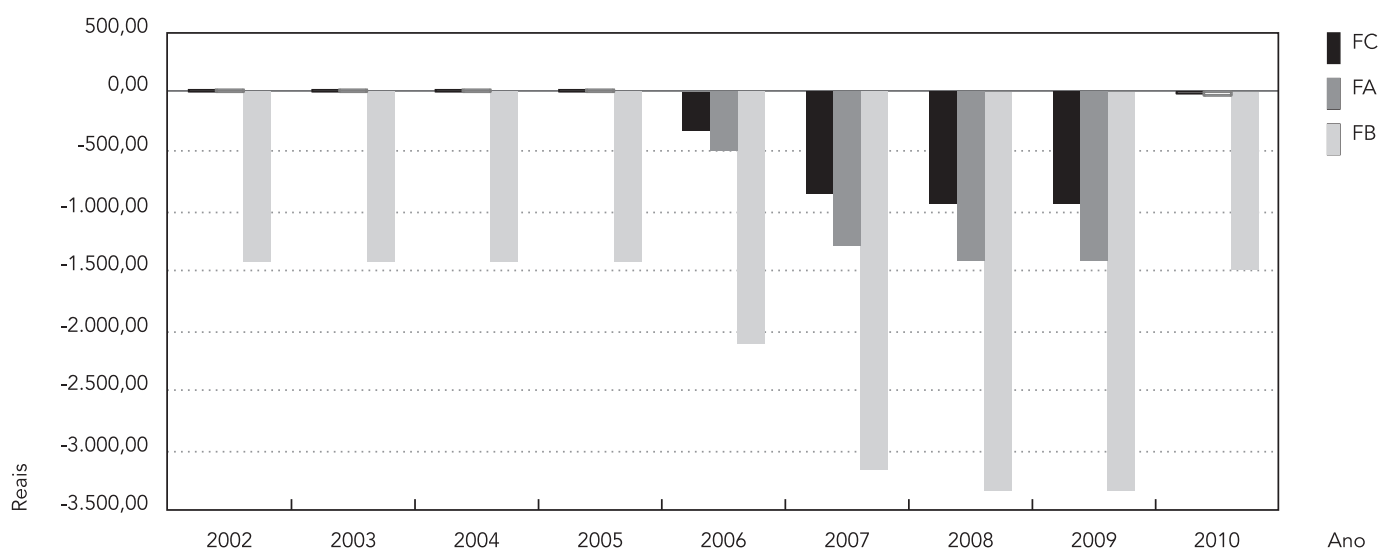

FC: fase crônica; FA: fase acelerada; FB: fase blástica.

Tabela 2

Valor repassado pelo Ministério da Saúde e gasto hospitalar mensais em Reais (R\$) nas fases crônica, acelerada e blástica, entre 2002 e 2010.

\begin{tabular}{ccccccc}
\hline & \multicolumn{2}{c}{ Fase crônica } & \multicolumn{2}{c}{ Fase acelerada } & \multicolumn{2}{c}{ Fase blástica } \\
& Repasse & Gasto & Repasse & Gasto & \multicolumn{2}{c}{ Repasse } \\
\hline 2002 & $4.067,00$ & $4.050,00$ & $6.092,00$ & $6.075,00$ & $6.678,50$ & $8.100,00$ \\
2003 & $4.067,00$ & $4.050,00$ & $6.092,00$ & $6.075,00$ & $6.678,50$ & $8.100,00$ \\
2004 & $4.067,00$ & $4.050,00$ & $6.092,00$ & $6.075,00$ & $6.678,50$ & $8.100,00$ \\
2005 & $4.067,00$ & $4.050,00$ & $6.092,00$ & $6.075,00$ & $6.678,50$ & $8.100,00$ \\
2006 & $4.067,00$ & $4.392,00$ & $6.092,00$ & $6.588,00$ & $6.678,50$ & $8.784,00$ \\
2007 & $4.067,00$ & $4.920,00$ & $6.092,00$ & $7.380,00$ & $6.678,50$ & $9.840,00$ \\
2008 & $4.067,00$ & $5.004,00$ & $6.092,00$ & $7.506,00$ & $6.678,50$ & $10.008,00$ \\
2009 & $4.067,00$ & $5.004,00$ & $6.092,00$ & $7.506,00$ & $6.678,50$ & $10.008,00$ \\
2010 & $4.067,00$ & $4.081,20$ & $6.092,00$ & $6.121,80$ & $6.678,50$ & $8.162,40$ \\
\hline
\end{tabular}

Conforme evidenciado em outras áreas, como no caso dos antirretrovirais (ARV), o acesso expandido pode ser considerado, na prática, o verdadeiro lançamento do medicamento, pois geralmente ao final do programa o medicamento já estará incorporado no sistema de saúde 11. A ampliação do "uso humanitário" de um medicamento mediante um programa de acesso expandido não resolve a questão da antecipação do acesso, ao contrário: introduz o medicamento, iniciando e assegurando o mercado.
O aumento do número de usuários em tratamento no HC-UFPR entre 2002 e 2010 reflete o encaminhamento destes usuários para os centros de referência, alavancando a organização desta área da hematologia. Em 2011, em decorrência da descentralização do serviço e encaminhamento deles para outros centros, de acordo com a regionalização do sistema de saúde, notase uma queda no número de usuários. O código de transação dificultou os encaminhamentos, represando os usuários para fora do HC-UFPR. 
Figura 2

Diferença (em Reais) entre o valor repassado pelo Ministério da Saúde e o valor pago na compra do mesilato de imatinibe para leucemia mieloide crônica por ano, de 2002 a 2010, no Hospital de Clínicas da Universidade Federal do Paraná (HC-UFPR), Brasil. Valores corrigidos pelo Índice Nacional de Preços ao Consumidor Amplo (IPCA).

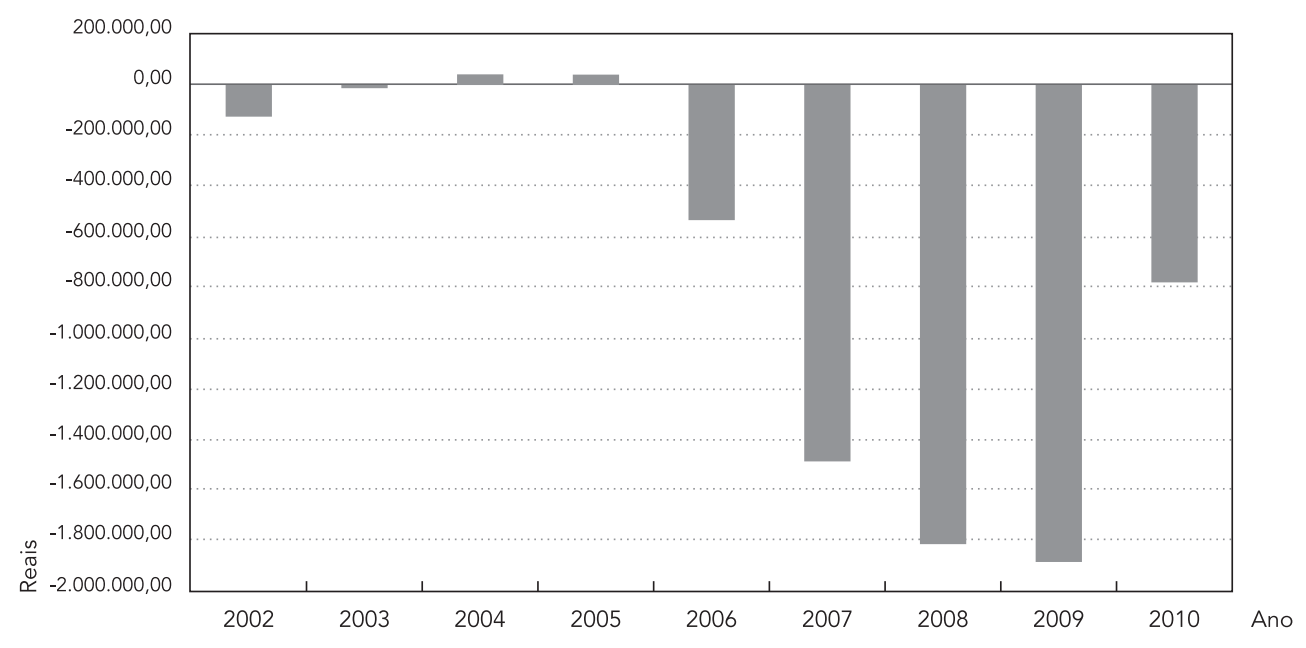

Figura 3

Variação do custo de tratamento por mês em leucemia mieloide crônica (fase crônica) no Hospital de Clínicas da Universidade Federal do Paraná (HC-UFPR), Brasil, de 2002 a 2011.

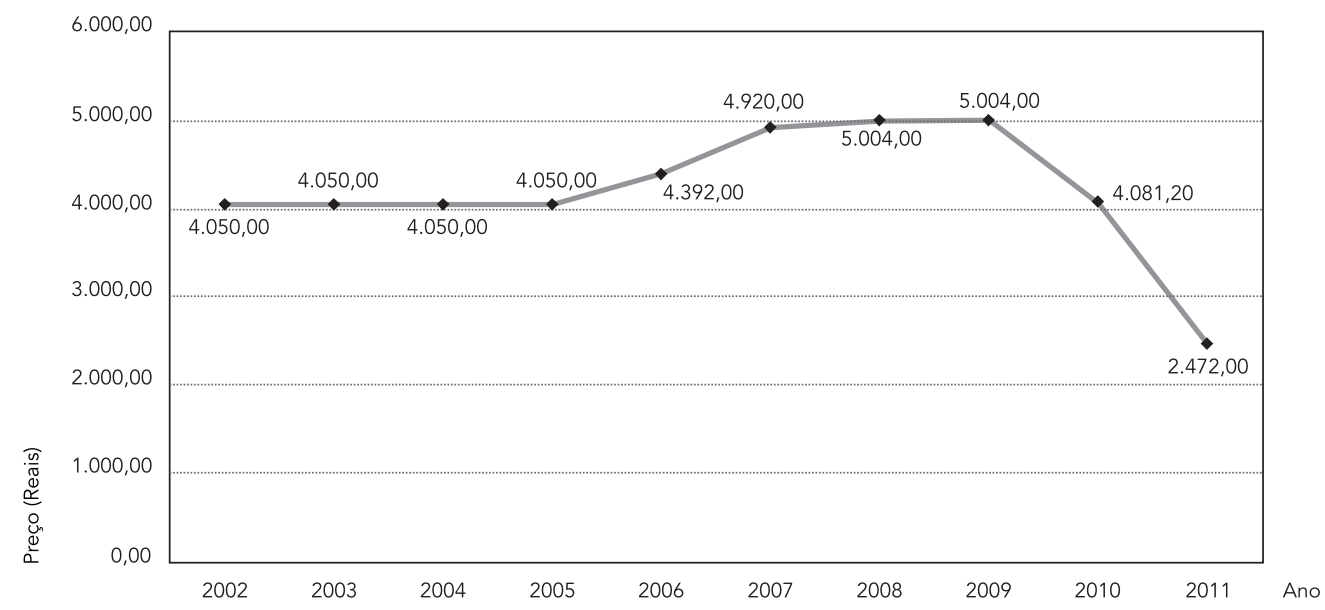

O gasto hospitalar na compra do medicamento e o repasse financeiro do Ministério da Saúde para o HC-UFPR apresentaram crescimento ao longo dos anos, sendo que o primei- ro aumentou bem mais que o segundo. A análise dos dados do Departamento de Informática do SUS (DATASUS. Produção ambulatorial. http://www2.datasus.gov.br/DATASUS/index. 
php?area $=0202 \& V O b j=h t t p: / /$ tabnet.datasus . gov.br/cgi/deftohtm.exe?sia/cnv/pa, acessado em 10/Nov/2012) no mesmo período evidenciou a ocorrência da mesma tendência de endividamento hospitalar no nível nacional. O aumento do valor pago para o tratamento de LMC não diz respeito a uma atualização da tabela SUS de APAC, mas está relacionado com o aumento do número de usuários no nível nacional e o investimento total por parte do governo.

Como o Ministério da Saúde, na época, não comprava diretamente o produto, mas só repassava recursos na forma de procedimentos de alta complexidade, houve transferência da responsabilidade do financiamento para os centros de tratamento, já que havia uma defasagem significativa entre o ressarcimento e o preço real do medicamento. $\mathrm{O}$ valor do produto inicialmente era equivalente ao valor repassado, mas, com o passar dos anos, o Ministério da Saúde não autorizou aumento da APAC da mesma forma como autorizou o aumento do preço do medicamento. $\mathrm{O}$ repasse era aquém do necessário e, nesse período, para compensar parte dessa defasagem que havia entre o valor pago e o valor recebido, a indústria farmacêutica fornecia uma quantidade de medicamento como bonificação, apesar dos atrasos no pagamento de seis meses até um ano, algumas vezes.

O fornecimento do mesilato de imatinibe por meio de doação, assim como o programa de acesso expandido, tem a função de criar demanda e fazer com que se mantenha a distribuição pública do medicamento. Na relação com o governo, que é o comprador majoritário no Brasil, a indústria utiliza essas e outras táticas de aproximação, relacionamento e "parcerias comerciais" 11.

Entre 2002 e 2005, para o tratamento da fase crônica e da fase acelerada, não havia problemas com o ressarcimento do Ministério da Saúde, o déficit é percebido somente para os poucos tratamentos em fase blástica, não impactando tanto no orçamento total do hospital. O ano de 2006 marca o início do endividamento do HCUFPR com a compra do mesilato de imatinibe, que quadruplica em três anos, passando de $\mathrm{R} \$$ 437.410,50 em 2006 para $\mathrm{R} \$ 1.781 .302,50 \mathrm{em}$ 2009. Em meados de 2010 ocorre uma redução progressiva do preço até abril de 2011, quando o Ministério da Saúde passa a financiar e distribuir o medicamento para os estados, municípios e centros de referência para o tratamento. Com a compra centralizada, além da redução do preço pago pelo medicamento, esses gastos deixaram de ser do HC-UFPR e passaram a ser de total responsabilidade do Ministério da Saúde. A dívida referente à compra do mesilato de imatinibe parou de aumentar, entretanto, esse valor acumu- lado ao longo dos anos nunca foi ressarcido pelo Ministério da Saúde.

Essa realidade de endividamento não é exclusividade do HC-UFPR. De acordo com a Associação Nacional dos Dirigentes das Instituições Federais de Ensino Superior (ANDIFES), em 2007, a dívida acumulada dos hospitais universitários era de R $\$ 440$ milhões. Pelos dados do Ministério da Educação (MEC), esses hospitais federais acumulavam um déficit de $\mathrm{R} \$ 30$ milhões por ano, levando à dívida de $\mathrm{R} \$ 425$ milhões em 2007. Esse déficit dizia respeito, principalmente, à defasagem na tabela de procedimentos e ao pagamento de salários de trabalhadores contratados, para cobrir a falta de servidores 12,13. Em um estudo envolvendo oito hospitais do Rio de Janeiro, foram constatados obstáculos de origem interna e externa, que contribuíam para a situação de precariedade do serviço. Dentre as questões externas, destaca-se a escassez de recursos 4 .

\section{Estratégia para garantir mercado}

O Glivec (Novartis, São Paulo, Brasil) - nome comercial do mesilato de imatinibe - teve como sede de suas pesquisas iniciais a University of Pennsylvania em 1960, com testes financiados principalmente pelo National Institutes of Health (NIH) dos Estados Unidos. A Novartis, que surge em 1996 da fusão entre Ciba-Geigy e Sandoz, teve participação reduzida nos gastos das pesquisas iniciais, alcançando aproximadamente $10 \%$ do investimento total. Esse conhecimento científico de décadas foi licenciado para o setor privado e os cientistas da indústria adaptaram a pesquisa para uso em diferentes situações clínicas $8,14,15,16,17$.

O medicamento teve sua patente reconhecida no Brasil pelo sistema pipeline, previsto na legislação de propriedade industrial aprovada em 1996. Embora essa lei tenha previsto o reconhecimento de patentes no setor farmacêutico apenas a partir de maio de 1997, o mecanismo do pipeline possibilitou o depósito de pedidos no setor farmacêutico entre maio de 1996 e maio 1997, desde que tivesse depósito no país de origem, não estivesse sendo comercializado em lugar algum e não houvesse esforços locais para a exploração da invenção. Foram aceitos pedidos fora do período de prioridade, não houve exame técnico no Brasil dos requisitos de patenteabilidade e a vigência das patentes concedidas foi contada a partir da data do primeiro depósito no país de origem. Isso significou que pedidos de patente cuja invenção já estava em domínio público ficassem sob o monopólio do detentor da patente no Brasil, restringindo a aquisição de 
versões genéricas mais baratas disponíveis no mercado internacional 18,19,20.

No Brasil, a Novartis ingressou com uma ação na justiça federal para ampliação da vigência da patente do mesilato de imatinibe para vinte anos a partir da data de registro na União Europeia, que aconteceu em 25 de março de 1993. Esse pedido foi negado pelo Supremo Tribunal de Justiça, que antecipou o fim da patente do medicamento tendo como base o primeiro depósito feito na Suíça em 03 de abril de 1992. A diferença de prazos para o fim do termo da patente tem grande relevância, porque a extensão da patente mobiliza grande quantidade de recursos, além de abrir precedentes para o prolongamento do monopólio em outros casos 19

É inegável o impacto positivo do mesilato de imatinibe no aumento da sobrevida e da qualidade de vida das pessoas em tratamento de LMC. Contudo, há que se olhar de forma crítica as estratégias utilizadas pela indústria farmacêutica para assegurar lucros sólidos, já que os hospitais não poderiam deixar de fornecer a medicação para usuários já em uso.

Analisando os dados financeiros e a magnitude da dívida acumulada por um dos maiores centros de tratamento de LMC no Brasil, o HCUFPR, é possível perceber que a sustentabilidade no fornecimento do mesilato de imatinibe foi ameaçada. Sem uma resposta governamental que mudasse os parâmetros de negociação com a indústria farmacêutica, o acesso ao tratamento ficaria prejudicado.

De forma análoga, no enfrentamento da epidemia de AIDS no Brasil, a incorporação de medicamentos ARV patenteados significou um aumento do gasto público, levantou questionamentos sobre a sustentabilidade da política de acesso universal a medicamentos e requereu respostas governamentais para redução de preços, tais como negociação de preços com as empresas detentoras das patentes, ameaça e uso da licença compulsória, entre outros 21,22. O dilema da incorporação de tecnologia e da situação de monopólio requer melhor planejamento sobre como assegurar o acesso, não só do ponto de vista da disponibilidade, mas também da sustentabilidade financeira, que é assegurada quando os preços são acessíveis.

Essa situação ensejou, no início de 2010, negociações do governo com a indústria farmacêutica a fim de obter redução do preço do medicamento. Após cinco meses, foi acertada uma redução progressiva de até $51 \%$ no preço do comprimido do mesilato de imatinibe com a compra centralizada pelo Ministério da Saúde, e posterior distribuição pelas secretarias de saúde aos centros de tratamento 7 . No entanto, a redução alcançada por meio da centralização da compra e negociação de preço requer uma análise mais detalhada de uma série histórica de preços praticados pela empresa 11 . Tal como observado na Figura 3, a redução alcançada em 2010 foi precedida de um aumento de $23,56 \%$ (valor não corrigido pelo Índice Nacional de Preços ao Consumidor Amplo - IPCA) no período de 2005 a 2010, quando a demanda pelo medicamento só aumentou.

\section{Estratégias governamentais para redução de preços}

O preço dos medicamentos é influenciado por uma gama de fatores, entre eles: pesquisa e desenvolvimento de novas substâncias, grau de inovação, potencial terapêutico, regulação, poder de demanda do mercado, entrada de genéricos, competição, marketing farmacêutico e proteção patentária 1,23,24. A incorporação da maioria das tecnologias acontece de forma complementar, não substitutiva, por isso esse processo acaba gerando aumento dos custos e gastos governamentais 1 . Dessa forma, o aumento no suprimento de tecnologias em saúde impacta significativamente nos gastos em saúde e compromete a sustentabilidade financeira do SUS 25,26.

Os preços da maioria dos medicamentos patenteados, via de regra, não têm relação direta com o custo de produção, refletem o seu valor de mercado. O poder de decisão e fixação de preço desses medicamentos está nas mãos da indústria farmacêutica e diz respeito a quanto o governo brasileiro pode pagar 11. Em geral, a conduta da indústria é de aferir o maior preço possível em um mercado, de acordo com esse contexto em particular 24

AANVISA, por meio da Câmara de Regulação do Mercado de Medicamentos (CMED), estabelece critérios para classificar os medicamentos e assim definir o teto do preço a ser praticado no mercado 27. Adicionalmente, a ANVISA também tem responsabilidade no exame dos requisitos de patenteabilidade para o setor farmacêutico no Brasil. Essa função atribuída à agência no processo de exame dos pedidos de patentes farmacêuticas foi prevista por meio da emenda à lei de propriedade industrial aprovada em 200120,28 .

Apesar das regulações propostas, o preço do medicamento continuou aumentando ao longo do tempo (Figura 3), tendo sido necessárias estratégias governamentais adicionais para redução do preço e manutenção da expansão do acesso ao tratamento no SUS. O aumento do preço foi possível em função da barreira patentária, que excluía concorrentes do mercado. 
Conforme discutido anteriormente, a primeira iniciativa governamental para redução do preço do mesilato de imatinibe foi a centralização da compra e negociação com a indústria em 2010, que alcançou considerável redução de preço. Outra estratégia governamental é a produção local de medicamentos.

Nos últimos trinta anos, o Brasil tornou-se capaz de produzir muitos produtos para a saúde, mas ainda há dependência quando se fala da necessidade de importação para obter novas tecnologias em saúde 29 . O fortalecimento da produção pública de medicamentos vem sendo considerada uma possível estratégia para reduzir os preços, garantir acesso aos usuários do SUS e diminuir a dependência do país 30,31,32. Desde 2008, os laboratórios farmacêuticos oficiais estão sendo considerados centrais nas políticas industriais editadas pelo governo, as quais têm tido como um dos seus objetivos a internalização da produção de produtos estratégicos para o SUS 33,34.

Como parte da atual política industrial e com o fim da vigência da patente do mesilato de imatinibe em 2012, o medicamento passou a ser objeto de uma Parceria para o Desenvolvimento Produtivo (PDP) entre dois laboratórios oficiais (Instituto de Tecnologia em Fármacos - Farmanguinhos e Instituto Vital Brazil) e cinco indústrias privadas nacionais (EMS, Laborvida, Cristália, Alfa Rio e Globe Química), gerando uma economia referida de $\mathrm{R} \$ 337$ milhões 35,36 . As PDP têm o objetivo de transferência de tecnologia em um período de cinco anos, a fim de estimular a produção totalmente nacional de medicamentos de alta complexidade 37 . Ficou fora do escopo da presente pesquisa a análise dos preços do produto produzido localmente e o aprofundamento sobre a importância desta estratégia.

\section{Considerações finais}

Informações precisas, confiáveis e atualizadas constituem ferramentas para o enfrentamento de problemas de saúde 38. A sistematização da história do mesilato de imatinibe, em uma instituição de ensino e pesquisa, apresenta grande valor em tornar públicas informações guardadas em arquivos e na memória, e correlacionar discussões sobre os gastos em saúde, propriedade intelectual, políticas e acesso a medicamentos. As dificuldades na sistematização dos dados dis- persos nos setores do hospital e no resgate da memória constituem limitações deste estudo. No entanto, as análises mantiveram-se confiáveis e coerentes com a realidade.

A sistematização dos dados e informações sobre a incorporação do mesilato de imatinibe no HC-UFPR constituiu a análise de um caso que pode ser ampliado para a compreensão deste processo nacionalmente.

O processo de endividamento do HC-UFPR em função da compra do mesilato de imatinibe, ao longo do período estudado, se deu não só pelo aumento da demanda, mas também em virtude da incompatibilidade entre o aumento do preço do medicamento e o valor da APAC repassado. O estudo mostrou que o caminho da incorporação do medicamento por um hospital universitário teve como pano fundo abordagens tanto da empresa como do governo.

De um lado, a indústria farmacêutica multinacional fez uso de duas estratégias: a primeira foi a introdução do uso do mesilato de imatinibe não mediada pela compra, com o acesso expandido e doações; a segunda, foi a proteção patentária do medicamento, assegurando o poder para definir preços, o que possibilitou o aumento do preço ao longo do tempo. De outro, o governo herdou os custos da continuidade de um tratamento, não regulou o aumento do preço, provocou o endividamento hospitalar pela diferença entre custo e APAC, centralizou a compra, negociou o preço e finalmente optou pela produção local.

As respostas para redução do preço neste caso foram, portanto, a centralização da compra e a nacionalização da produção por meio da PDP. Seria necessário aprofundar em futuras pesquisas se a opção por essas estratégias são as que promovem maiores reduções de preços para o governo.

Mesmo que a proposta de nacionalização da produção por meio das PDP seja ampliar o acesso, há que considerar potenciais tensões entre os setores público e privado, e também assegurar o comprometimento com as políticas de saúde e melhoria das condições de vida da população. Este trabalho também servirá como subsídio para outras pesquisas que aprofundem o conhecimento sobre o uso de recursos gastos em medicamentos no Brasil e avaliem as PDP como estratégia de produção local e promoção do acesso a novas tecnologias. 


\section{Resumen}

Los gastos en medicamentos representan una gran proporción del presupuesto de salud, por lo que la producción de conocimiento sobre el uso de estos recursos es esencial en la toma de decisiones en salud pública y la mejora de la atención farmacéutica. Este estudio tuvo como objetivo analizar el proceso de endeudamiento en un hospital universitario de alta complejidad, debido al aumento de los gastos en la adquisición de mesilato de imatinib. A través del análisis de los documentos y registros en el Sistema de Información Hospitalaria (SIH) entre 2002 y 2010, se realizó un estudio descriptivo. A partir de la incorporación del medicamento, se mapearon las estrategias de la industria farmacéutica y del gobierno, así como las respuestas del gobierno para reducir el precio. La sistematización y publicación de la información almacenada en los archivos y su memoria puede contribuir para el seguimiento de los resultados de los programas mantenidos por el Ministerio de Salud.

Costos de Hospital; Propiedad Intelectual;

Preparaciones Farmacéuticas

\section{Referências}

1. Lima EC, Sandes VS, Caetano R, Osório-de-Castro CGS. Incorporação e gasto com medicamentos de relevância financeira em hospital universitário de alta complexidade. Cad Saúde Colet (Rio J.) 2010; 18:551-9.

2. Departamento de Ciência e Tecnologia, Secretaria de Ciência e Tecnologia e Insumos Estratégicos, Ministério da Saúde. Avaliação de tecnologias em saúde: institucionalização das ações no Ministério da Saúde. Rev Saúde Pública 2006; 40:743-7.

3. Vieira FS. Gasto do Ministério da Saúde com medicamentos: tendência dos programas de 2002 a 2007. Rev Saúde Pública 2009; 43:674-81.

\section{Colaboradores}

C. T. Scopel foi responsável pela concepção do estudo, coleta e análise dos dados, redação e revisão final. G. C. Chaves contribuiu com a organização do trabalho, discussão dos dados e revisão final.

\section{Agradecimentos}

À Unidade de Farmácia Hospitalar do Hospital de Clínicas da Universidade Federal do Paraná (HC-UFPR) pelo apoio científico. Aos setores de abastecimento e faturamento do HC-UFPR pela disponibilização dos dados numéricos. Aos residentes farmacêuticos pela compreensão e agradável companhia diária. À Claudia Garcia Serpa Osorio-de-Castro e à Maria Auxiliadora Oliveira pelos comentários construtivos no primeiro esboço do manuscrito.
4. Azevedo CS, Fernandes MIA, Carreteiro TC. Sob o domínio da urgência: a prática de diretores de hospitais públicos do Rio de Janeiro, Brasil. Cad Saúde Pública 2007; 23:2410-20.

5. Departamento de Assistência Farmacêutica e Insumos Estratégicos, Secretaria de Ciência, Tecnologia e Insumos Estratégicos, Ministério da Saúde. Perguntas frequentes sobre a distribuição do Glivec e do tratamento da leucemia mieloide crônica e do tumor de estroma gastrintestinal no âmbito do SUS. http://portal.saude.gov.br/portal/arquivos/ pdf/perguntas_respostas_mesilato_imatinibe_ set_2011.pdf (acessado em 10/Fev/2012). 
6. Ministério da Saúde. Portaria SAS/MS no 431, de 3 de outubro de 2001. Aprova o protocolo clínico e diretrizes terapêuticas: tratamento da leucemia mieloide crônica do adulto. Diário Oficial da União 2001; 5 out.

7. Instituto Nacional de Câncer. Ministério da Saúde negocia com indústria e reduz preço do Glivec em 51\%. http://www2.inca.gov.br/wps/wcm/ connect/comunicacaoinformacao/site/home/sa la_imprensa/releases $/ 2010 /$ ministerio_negocia_ com_industria_e_reduz_preco_do_glivec_em_51 (acessado em 18/Jun/2010).

8. Macedo A. A arte de cuidar e curar na leucemia mieloide crônica. São Paulo: Planmark; 2012.

9. Funke VAM. Terapia alvo com mesilato de imatinibe: um estudo de 98 pacientes com leucemia mieloide crônica [Dissertação de Mestrado]. Curitiba: Universidade Federal do Paraná; 2005.

10. Agência Nacional de Vigilância Sanitária. Resolução RDC MS/ANVISA no 26, de 17 de dezembro de 1999. Aprova regulamento, constante no anexo desta Resolução, destinado a normatizar a avaliação e aprovação de programas de acesso expandido somente de produtos com estudos de fase III em desenvolvimento no Brasil ou no país de origem e com programa de acesso expandido aprovado no país de origem, ou com registro do produto no país de origem. Diário Oficial da União 1999; 20 dez.

11. Scheffer MC. AIDS, tecnologia e acesso sustentável a medicamentos: a incorporação dos anti-retrovirais no Sistema Único de Saúde [Tese de Doutorado]. São Paulo: Universidade de São Paulo; 2008.

12. Pilotto B. Os hospitais universitários e sua crise. http://www.scribd.com/doc/36023434/Os-Hospi tais-Universitarios-e-Sua-Crise (acessado em 01/ Mai/2014).

13. Cislaghi JF. Hospitais universitários: presente caótico e futuro incerto. http://www.apufpr.org.br/ artigos/hus.pdf (acessado em 01/Mai/2014).

14. Dreifus C. Researcher behind the drug Gleevec. The New York Times 2009; 3 nov. http://www.ny times.com/2009/11/03/science/03conv.html? pagewanted $=1$ (acessado em 10/Fev/2011).

15. Angell M. A verdade sobre os laboratórios farmacêuticos. Rio de Janeiro: Record; 2009.

16. Azoulay P, Zivin JG, Li D, Sampat B. Public R\&D investments and private sector patenting: evidence from NIH funding rules. https://www.funginsti tute.berkeley.edu/sites/default/files/danielle\%20 paper.pdf (acessado em 06/Nov/2014).

17. Love J. R\&D costs for Gleevec. http://www.keion line.org/node/1697 (acessado em 17/Fev/2014).

18. Hasenclever L, Lopes R, Chaves GC, Reis R, Vieira MF. O instituto de patentes pipeline e o acesso a medicamentos: aspectos econômicos e jurídicos deletérios à economia da saúde. Rev Direito Sanit 2010; 11:164-88.

19. Superior Tribunal de Justiça. Patente do medicamento Glivec, do laboratório Novartis, vence em 2012. http://www.stj.gov.br/portal_stj/publica cao $/$ engine.wsp?tmp.area $=398 \&$ tmp.texto $=99586$ (acessado em 03/Jul/2012).
20. Brasil. Lei no 9.279, de 14 de maio de 1996. Regula direitos e obrigações relativos à propriedade industrial. Diário Oficial da União 1996; 15 mai.

21. Grangeiro A, Teixeira L, Bastos FI, Teixeira P. Sustentabilidade da política de acesso a medicamentos anti-retrovirais no Brasil. Rev Saúde Pública 2006; 40 Suppl:60-9.

22. Ford N, Wilson D, Chaves GC, Lotrowska M, Kijtiwatchakul K. Sustaining access to antirretroviral therapy in the less-developed world: lessons from Brazil and Thailand. AIDS 2007; 21 Suppl 4:S21-9.

23. Reis ALA, Bermudez JAZ, Oliveira MA. Effects of the TRIPS agreement on the access to medicines: considerations for monitoring drug prices. In: Bermudez JAZ, Oliveira MA, editors. Intellectual property in the context of the WHO TRIPS agreement: challenges for public health. Rio de Janeiro: Escola Nacional de Saúde Pública Sergio Arouca, Fundação Oswaldo Cruz; 2004. p. 99-115.

24. Reis ALA, Bermudez J. Aspectos econômicos: mercado farmacêutico e preços de medicamentos. In: Bermudez JAZ, Oliveira MA, Esher A, organizadores. Acceso a medicamentos: derecho fundamental, papel del estado. Rio de Janeiro: Escola Nacional de Saúde Pública Sergio Arouca, Fundação Oswaldo Cruz; 2004. p. 139-55.

25. Silva MT. Evaluación de tecnologías sanitárias: la experiencia en el ministerio de salud de Brasil. Rev Peru Med Exp Salud Pública 2011; 28:548-51.

26. Silva MT, Almeida RT, Gava CM, Galvão TF, Silva EMK, Santos VCC, et al. Brazilian health technology assessment bulletin: editorial process, dissemination strategies, critical appraisal, and initial impact. Int J Technol Assess Health Care 2012; 28:65-9.

27. Gerência de Avaliação Econômica de Novas Tecnologias, Agência Nacional de Vigilância Sanitária. Efeito da resolução CMED no 02/04 no processo de análise de preços de novos medicamentos. http://portal.anvisa.gov.br/wps/wcm/ connect/31cbde804e311d5694ddb7c09d49251b/ trabalho+balan\%C3\%A7o+resol+2.final.15. Jan.2013.pdf?MOD=AJPERES (acessado em 01/ Mai/2014).

28. Brasil. Lei no 10.196, de 14 de fevereiro de 2001. Altera e acresce dispositivos à Lei no 9.279, de 14 de maio de 1996, que regula direitos e obrigações relativos à propriedade industrial, e dá outras providências. Diário Oficial da União 2001; 16 fev.

29. Banta D, Almeida RT. The development of health technology assessment in Brazil. Int J Technol Assess Health Care 2009; 25 Suppl 1:255-9.

30. Gadelha CAG. A dinâmica do sistema produtivo da saúde: inovação e complexo econômico-industrial. Rio de Janeiro: Editora Fiocruz; 2012.

31. Oliveira EA, Labra ME, Bermudez J. A produção pública de medicamentos no Brasil: uma visão geral. Cad Saúde Pública 2006; 22:2379-89.

32. Magalhães JL, Antunes AMS, Boechat N. Laboratórios farmacêuticos oficiais e sua relevância para saúde pública do Brasil. Revista Eletrônica de Comunicação, Informação \& Inovação em Saúde 2011; 5:85-99. 
33. Ministério da Saúde. Portaria no 978, de 16 de maio de 2008. Dispõe sobre a lista de produtos estratégicos, no âmbito do Sistema Único de Saúde, com a finalidade de colaborar com o desenvolvimento do Complexo Industrial da Saúde, e institui a Comissão para Revisão e Atualização da referida lista. Diário Oficial da União 2008; 19 mai.

34. Ministério da Saúde. Portaria no 1.284, de 26 de maio de 2010. Altera o anexo à Portaria no 978/ GM/MS, de 16 de maio de 2008. Diário Oficial da União 2010; 27 mai.

35. Instituto Vital Brazil. Medicamentos produzidos. http:/ /www.vitalbrazil.rj.gov.br/medicamentos_ produzidos.html (acessado em 20/Out/2013).

36. Instituto de Tecnologia em Fármacos, Fundação Oswaldo Cruz. Acordo de produção. http:// www2.far.fiocruz.br/farmanguinhos/index. php?option=com_content $\&$ view $=$ article $\&$ id $=$ 482:farmanguinhos-assina-acordo-para-pro duzir-mesilato-de-imatinibe $\&$ catid=53:outras noticias\&Itemid=94 (acessado em 20/Out/2013).
37. Ministério da Saúde. Portaria MS/GM no 837, de 18 de abril de 2012. Define as diretrizes e os critérios para o estabelecimento das Parcerias para o Desenvolvimento Produtivo (PDP). Diário Oficial da União 2012; 27 abr.

38. Instituto Nacional de Câncer. ABC do câncer: abordagens básicas para o controle do câncer. Rio de Janeiro: Instituto Nacional de Câncer; 2011.

Recebido em 22/Mai/2014

Versão final reapresentada em 19/Ago/2014

Aprovado em 31/Out/2014 